\title{
Validation of Algorithms for the Estimation of Pulse Transit Time: Where do We Stand Today?
}

\author{
Theodoros G. Papaioannou, ${ }^{1,2}$ Orestis Vardoulis, ${ }^{1}$ and Nikolaos Stergiopulos ${ }^{1}$ \\ ${ }^{1}$ Laboratory of Hemodynamics and Cardiovascular Technology, Ecole Polytechnique Fédérale de Lausanne, Lausanne, \\ Switzerland; and ${ }^{2}$ Biomedical Engineering Unit, First Department of Cardiology, Hippokration Hospital, Medical School, \\ National and Kapodistrian University of Athens, Athens, Greece
}

(Received 6 February 2014; accepted 28 March 2014; published online 10 April 2014)

Associate Editor Dan Elson oversaw the review of this article.

Gaddum et al., ${ }^{2}$ recently published in Annals of Biomedical Engineering an interesting article seeking the optimal method for the estimation of arterial pulse transit time (TT) between two arterial sites. Accurate and precise TT estimation in vivo is a critical issue, because TT is one of the two parameters (together with distance measurement) needed for the calculation of pulse wave velocity (PWV).

PWV is the hallmark of the assessment of arterial stiffness in clinical practice. To date carotid-to-femoral PWV, a measure of segmental stiffness between ascending aorta and femoral artery, is recommended as the gold standard to evaluate arterial stiffening. ${ }^{3} \mathrm{PWV}$ is estimated by the ratio of the distance $(\mathrm{m})$ travelled by a pressure, velocity or distension wave between two arterial sites to the TT. It is not possible to measure TT in vivo by direct means and hence several computational algorithms have been proposed aiming to determine the time delay between two waves.

Gaddum et al..$^{2}$ aimed to determine the most reliable algorithm for TT estimation. Nevertheless, a clear distinction should be made among different analyses of velocity, pressure or distension waveforms. Since each wave is physiologically different from the other and also it is acquired by different techniques, it is likely that there is no single algorithm which will be equally reliable when applied to different waves.

Cross-correlation based techniques have been used for the processing of MRI-derived flow waveforms ${ }^{1}$ with positive results. Other algorithms are based on "least

Address correspondence to Theodoros G. Papaioannou, Biomedical Engineering Unit, First Department of Cardiology, Hippokration Hospital, Medical School, National and Kapodistrian University of Athens, Athens, Greece. Electronic mail: thepap@ med.uoa.gr squared errors" similarly to the study of Gaddum et al. ${ }^{2}$ It would be interesting to know what kind of "least squares" algorithm was used in this study ${ }^{2}$ (details are limited), in order to further evaluate or expand the use of this method, since several requirements should be met for its optimal application. Vardoulis et al. ${ }^{4}$ previously proposed a squared errors minimization method ("diastole patching" method) where a diastole patch (region) of proximal pressure waveform is scanned along the distal waveform, one time-step at a time. TT patch was then defined as the sum of the time-steps until the minimum of the squared differences is met. The signal was carefully set as the region that is centered in the diastolic minimum and spans two times the length between the diastolic minimum up to the point of the maximum first derivative. This choice was of crucial importance since beyond that point the signal is affected by the reflected waves.

In this study ${ }^{2}$ the tested least squares algorithm takes into account a region spanning from the diastolic minimum up to the peak systolic value of pressure. However, it has been shown that the systolic peak can be highly distorted by wave reflections, especially in diseased populations where the reflected waves return earlier due to high PWV. The inclusion of this region could result in unstable performance, especially at individuals with stiff arteries.

Also it should be highlighted that Gaddum et al. focused on analyzing waveforms of the aortic trunk. Although this is the best pathway to benchmark techniques, it still differs from standard clinical practice were waveforms are measured at carotid and femoral arteries. It would be essential to see how the proposed cross-correlation coefficient ${ }^{2}$ perform upon a set of carotid and femoral simulated waveforms, which definitely differ from aortic waveforms. 
A major contribution of this study ${ }^{2}$ is the in vivo evaluation of TT algorithms using arterial pressure waves recorded by applanation tonometry using SphygmoCor apparatus. Nevertheless, this device does not record carotid and femoral waveforms simultaneously and ECG gating is required to perform the subsequent TT calculations. It is quite unclear how "beat-to-beat analysis" can be applied in that case.

Up to date there is no standard protocol or consensus for the validation of algorithms for TT estimation, since there is no direct gold standard method for in vivo TT or PWV measurement. Mathematical models of the arterial tree are appropriate for in silico validation. Pressure or velocity waveforms can be reproduced under various conditions while the "true" value of wave speed can be analytically calculated. However, these models require their own validation. Hence, it is very essential to use accurate models that: (a) yield realistic hemodynamic data and (b) represent reliably pathophysiological cardiovascular changes and responses to different stimuli. Nonetheless, it is imperative to compare the model-derived pressure and velocity waves with in vivo recordings. The model of this study ${ }^{2}$ has been "validated" by using in vitro data and thus in vivo studies should be also performed to further prove its accuracy.

PWV is an independent predictor of cardiovascular events and all-cause mortality in several populations and recent meta-analyses have verified this evidence. Nonetheless, it should be corrected that the metaanalysis referenced by Gaddum et al., (\#22) examined the prognostic value of central blood pressures and not PWV. Probably the authors intended to cite another meta-analysis by the same group. ${ }^{5}$
Indisputably the accuracy and precision of in vivo TT estimation remain the Achilles' heel of PWV measurement. A more reliable TT estimation could result to an enhancement of the predictive value of PWV, especially in cases where PWV currently fails to predict cardiovascular risk. Mathematical arterial models can serve this purpose by validating, in silico, new algorithms but such validation protocols should be first standardized.

\section{REFERENCES}

${ }^{1}$ Fielden, S. W., B. K. Fornwalt, M. Jerosch-Herold, R. L. Eisner, A. E. Stillman, and J. N. Oshinski. A new method for the determination of aortic pulse wave velocity using cross-correlation on 2D PCMR velocity data. J. Magn. Reson. Imaging 27:1382-1387, 2008.

${ }^{2}$ Gaddum, N. R., J. Alastruey, P. Beerbaum, P. Chowienczyk, and T. Schaeffter. A technical assessment of pulse wave velocity algorithms applied to non-invasive arterial waveforms. Ann. Biomed. Eng. 41:2617-2629, 2013.

${ }^{3}$ Laurent, S., J. Cockcroft, L. Van Bortel, P. Boutouyrie, C. Giannattasio, D. Hayoz, B. Pannier, C. Vlachopoulos, I. Wilkinson, and H. Struijker-Boudier. Expert consensus document on arterial stiffness: methodological issues and clinical applications. Eur. Heart J. 27:2588-2605, 2006.

${ }^{4}$ Vardoulis, O., T. G. Papaioannou, and N. Stergiopulos. Validation of a novel and existing algorithms for the estimation of pulse transit time: advancing the accuracy in pulse wave velocity measurement. Am. J. Physiol. Heart Circ. Physiol. 304:H1558-H1567, 2013.

${ }^{5}$ Vlachopoulos, C., K. Aznaouridis, and C. Stefanadis. Prediction of cardiovascular events and all-cause mortality with arterial stiffness: a systematic review and meta-analysis. $J$. Am. Coll. Cardiol. 55:1318-1327, 2010. 